\title{
Achievements of the ELISE test facility in view of the ITER NBI
}

\author{
B. Heinemann, D. Wünderlich, W. Kraus, F. Bonomo, U. Fantz, M. Fröschle, I. Mario, R. Riedl, C. Wimmer
}

Max Planck Institute for Plasma Physics, Boltzmannstr. 2, 85748 Garching, Germany

Neutral Beam Injection (NBI) for ITER shall deliver in total 33 MW heating power to the plasma with two injectors at a beam energy of $1 \mathrm{MV}$. Taking neutralization efficiency and all losses along the beam path into account a negative ion current density of $329 \mathrm{~A} / \mathrm{m}^{2}\left(\mathrm{H}^{-}\right.$for $1000 \mathrm{~s}$ ) and $286 \mathrm{~A} / \mathrm{m}^{2}$ ( $\mathrm{D}^{-}$for $3600 \mathrm{~s}$ ) has to be extracted from each ion source $\left(\right.$ size $1 \times 2 \mathrm{~m}^{2}$ ) with a beam uniformity $>90 \%$. For the optimization of the radio frequency driven (RF) negative ion source and its extraction system the ELISE test facility has been set up at IPP as a size scaling experiment with a source area of $0.9 \times 1 \mathrm{~m}^{2}$ and an extraction area of $0.1 \mathrm{~m}^{2}$.

Since its experimental start in 2013 continuous progress was achieved improving the system technically for long pulse operation at high RF power (total $300 \mathrm{~kW}$ for 4 drivers) as well as approaching ITER's physics requirements for the ion beam. For hydrogen $\sim 90 \%$ of the required ion current density can be extracted for long pulses $(1000 \mathrm{~s})$; the accelerated current density even exceeded the ITER target. For deuterium operation the main challenge is the amount, temporal in-stability and spatial asymmetry of co-extracted electron current which limit the applicable RF power and the source performance.

Shaping the magnetic field topology and controlling the electric fields inside the source together with an improved caesium conditioning procedure have been identified as possible tools to improve the source performance.

Keywords: Neutral Beam Injection, ITER, ELISE, negative ion source, radio frequency source

\section{Introduction}

The Neutral Beam Injection (NBI) system at ITER [1] is based on large RF (radio frequency) driven ion sources $\left(0.9 \times 2.0 \mathrm{~m}^{2}\right)$. To consolidate the source design the test facility ELISE (Extraction from a Large Ion Source Experiment) was set up at IPP as an intermediate step between the prototype RF source $\left(0.3 \times 0.6 \mathrm{~m}^{2}\right)$ $[2,3]$ and the full ITER size source. The ELISE ion source has the same width, but half the height of the ITER source $\left(0.9 \times 1 \mathrm{~m}^{2}\right)$ and an extraction area of $0.1 \mathrm{~m}^{2}$. Its goal is to identify and solve at an early stage any physical or technical issues which could prevent reaching the ITER target and to provide input for the operation of the EU Neutral Beam Test Facility (NBTF) in Padua [4], hosting SPIDER and MITICA experiments.

The aim of ELISE is to demonstrate the following ITER requirements: accelerated current densities of $200 \mathrm{~A} / \mathrm{m}^{2} \mathrm{D}^{-}$for $3600 \mathrm{~s}$ and $230 \mathrm{~A} / \mathrm{m}^{2} \mathrm{H}^{-}$for $1000 \mathrm{~s}$ at a source filling pressure $0.3 \mathrm{~Pa}$. As $30 \%$ stripping losses of negative ions by collisions with the background gas have been calculated inside the extraction system of ITER, this requires negative ion current densities to be extracted out of the ion source of $286 \mathrm{~A} / \mathrm{m}^{2} \mathrm{D}^{-}$and $329 \mathrm{~A} / \mathrm{m}^{2} \mathrm{H}^{-}$ . Furthermore, the co-extracted electron current has to be lower than the extracted ion current and the uniformity of the large beam must be better than $90 \%$.

The ELISE plasma is generated in 4 cylindrical drivers by inductive coupling of the RF power to the plasma (two $1 \mathrm{MHz}$ RF generators, max power $75 \mathrm{~kW} /$ driver). The three-stage extraction system has a similar aperture pattern and geometry as ITER (grouped in 8 arrays of $5 \times 16$ apertures, $\varnothing 14 \mathrm{~mm}$ ). Each stage has a top and a bottom grid segment which are electrically insulated to diagnose vertical current non-uniformities. An overview of the ELISE test facility is shown in Fig. 1 and present-

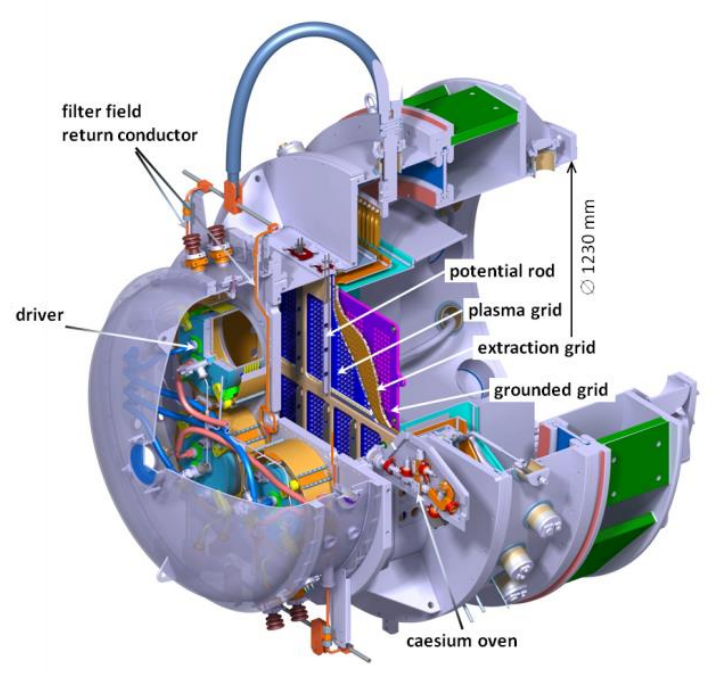

Fig. 1: Overview of the ELISE ion source ed in detail in $[5,6]$.

The negative ions are mainly produced by surface conversion of atoms and positive ions on the plasma grid (PG) surface which is covered by a thin caesium (Cs) layer in order to reduce the work function. The $\mathrm{Cs}$ is evaporated into the source volume continuously during an experimental day by two Cs-ovens and redistributed during plasma operation and during beam extraction. It is one of the main challenges to produce and maintain a favorable Cs coverage of the PG (source conditioning, [7]).

While plasma operation at ELISE is feasible continuously, beam extraction $(\leq 12 \mathrm{kV})$ and acceleration (total voltage $60 \mathrm{kV}$ ) is possible presently only in pulsed mode $(9.5 \mathrm{~s} / 150 \mathrm{~s})$ due to limitations of the HV-power supply. As this pulsed operation is different from ITER operation, a steady state power supply is going to be procured. 


\section{Source improvements}

For reliable source operation at high RF power several technical improvements were stepwise implemented at ELISE: i) replacing the two self-excited oscillators (180 kW each) by solid state amplifiers $(150 \mathrm{~kW})$ to avoid matching problems and frequency flips, ii) improved cooling of several RF-heated components, iii) avoiding external RF breakdowns at the coils by introducing a $2 \mathrm{~mm}$ gap between coil and driver and modifying the triple point geometry. More details on these improvements can be found in [8].

Furthermore achieving high ion currents for long pulses is especially challenging because of the amount and temporal increase of the co-extracted electron current, in particular in deuterium operation. The electron current is reduced by the magnetic filter field in front of the PG and a bias potential between the PG and source walls. However, this filter field causes cross-B-drifts in the plasma and a significantly higher electron current onto the top EG segment. As deuterium requires a higher filter field, this asymmetry is even more pronounced and the power interlock on the top EG segment $(125 \mathrm{~kW})$ limits the applicable RF power. A possible way to reduce the electron currents and make the ratio between top and bottom current more symmetric was found by adding permanent magnets along the side walls [9] and by introducing "vertical potential rods" in front of the PG [10]. The latter are cooled plates (30 mm wide) installed in 3 columns between the beamlet groups (fig. 1). By keeping these rods at PG potential and both biased positively against the source the power load on the EG by co-extracted electrons could be kept low enough, such that the maximum available RF power of $300 \mathrm{~kW}$ could be applied in hydrogen operation. The disadvantage was a longer Cs-conditioning time, but once achieved the lower plasma load reduced also depletion of Cs from the PG. Recently also the MITICA/ITER-like filter field (FF) has been simulated on ELISE and gives indication for improvement as described in chapter 4.

\section{Results achieved}

Since the start of experiments in 2013 continuous progress could be achieved at ELISE for both isotopes as shown in Fig. 2 for short pulses (9.5 s) and medium long pulses (plasma time $\geq 300$ s with $\geq 3$ beam blips).

In hydrogen it was possible in 2018 with the above mentioned improvements to perform pulses with accelerated current densities of $251 \mathrm{~A} / \mathrm{m}^{2}$ which even exceed the ITER requirement $\left(230 \mathrm{~A} / \mathrm{m}^{2}\right)$. This was achieved with extracted current densities below the ITER requirement as the stripping losses at ELISE are much smaller $(\sim 10 \%)$ than the values calculated for ITER [11]. Increasing the extracted currents at ELISE is only limited by the available HV power supply (max. $30 \mathrm{~A}$ ) and $\mathrm{RF}$ power supplies and it is estimated that an RF power of $85 \mathrm{~kW} /$ driver is sufficient to achieve also the extracted current densities (available at ITER: $100 \mathrm{~kW} /$ driver).

Also very promising results for long pulses $\geq 1000 \mathrm{~s}$ could be achieved in hydrogen at $0.3 \mathrm{~Pa}$ source filling pressure with an RF power of $74.5 \mathrm{~kW} /$ driver (Fig. 3). The total pulse length was $1200 \mathrm{~s}$ with stable ion cur-
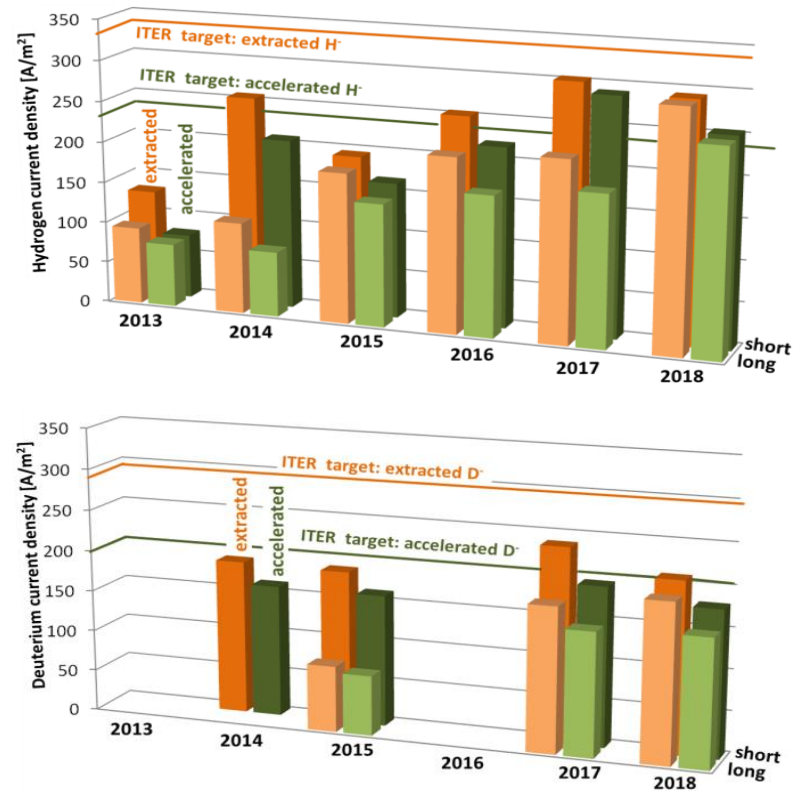

Fig. 2: Current densities achieved in hydrogen (top) and deuterium (bottom) for short pulses $(9.5 \mathrm{~s})$ and medium long pulses $(\geq 300$ s, with 3 beam blips). Electrically measured extracted current densities in orange, calorimetrically measured accelerated current densities in green.

rents during 6 beam blips over $1000 \mathrm{~s}$ ( $9.5 \mathrm{~s}$ per blip). A current density of $237 \mathrm{~A} / \mathrm{m}^{2}$ accelerated $\mathrm{H}^{-}$has been measured by water calorimetry of the beam dump as well as by infrared thermography, slightly above the ITER requirement. This result can be achieved by evaporating so much Cs into the source before the long pulse that the performance already starts to decrease, called "overconditioning" [12]. The initial $200 \mathrm{~s}$ plasma (preplasma) and first beam blip of the long pulse are considered to re-distribute the Cs inside the source beneficially.

The electron current densities do not reach a stable condition and increase over the pulse duration. This is explained by depletion of Cs from the PG surface by the plasma. However, the electron current is quite symmetric between the top and bottom half of the beam and stays over the pulse duration at a level which does not require reducing the RF power. On the right side of Fig. 3 the time trace of the third beam blip shows the Csconditioning dynamics during beam extraction. It is assumed that the backstreaming positive ions release Cs from the source back plate at energies high enough to overcome the positive bias potential of the PG (2-3 V).

Moreover in 2018 it was possible for the first time to sustain the conditioning status of the source over the whole day and to perform similar pulses in series. Fig. 4 presents five $1200 \mathrm{~s}$ pulses at $73 \mathrm{~kW}$. All pulses show a similar behavior with lower current densities for the first beam blip. For the second pulse only a plasma phase of $200 \mathrm{~s}$ was tested before the first beam blip, which had almost the same effect. For the third pulse the source filling pressure was raised to $0.4 \mathrm{~Pa}$ which increases the ion current density slightly by $\sim 4 \%$ and simultaneously decreases the co-extracted electron current density by $\sim 40 \%$. In general the experiments showed that a slightly higher source filling pressure eases source operation due to reduced amount of co-extracted electrons, in particu- 


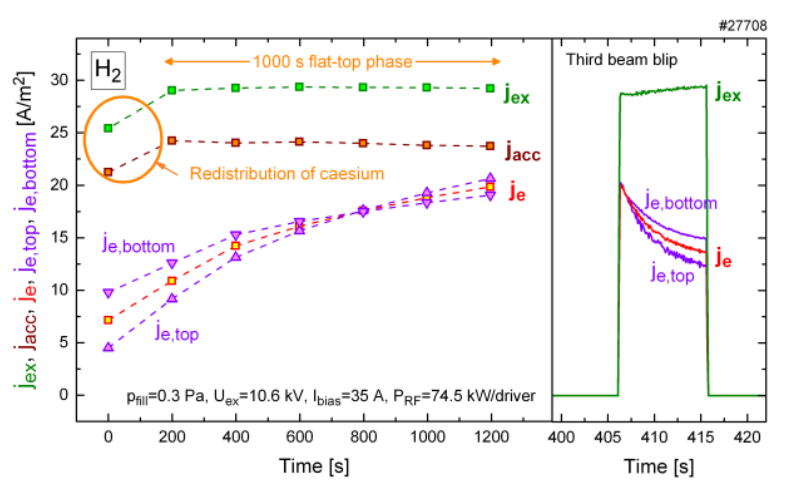

Fig. 3: Left: best $1200 \mathrm{~s}$ pulse in hydrogen with a $1000 \mathrm{~s}$ flat top ion current density with 8 beam blip at $74.5 \mathrm{~kW} /$ driver, $0.3 \mathrm{~Pa}, U_{e x}=10.6 \mathrm{kV}$. Right: time trace of the third beam blip as an example of all blips.

lar for high power operation. Further detailed modelling of the expected stripping losses at ITER should be performed to evaluate possible margins in the source pressure requirement of $0.3 \mathrm{~Pa}$.

In deuterium operation the co-extracted electron current and its top-bottom-asymmetry is in general much higher and limiting the applicable RF power. For long pulses current densities of $194 \mathrm{~A} / \mathrm{m}^{2}$ (extracted) and $152 \mathrm{~A} / \mathrm{m}^{2}$ (accelerated) could be achieved [10], but less deuterium campaigns were done so far. Modifications of the magnetic filter field strength and source potentials are promising tools for the suppression of these coextracted electrons.

Beam diagnostic is done on ELISE by a diagnostic calorimeter equipped with thermocouples, water calorimetry and IR imaging, and by beam emission spectroscopy (BES) with 20 lines of sight $[6,13]$. As an example the IR images of the $1^{\text {st }}, 3^{\text {rd }}$ and $7^{\text {th }}$ beam blip of the best pulse of Fig. 3 are shown in Fig. 5. Beside the lower ion current density of the $1^{\text {st }}$ blip also a strong top-bottomasymmetry is visible, possibly caused by the Cs distribution. Beam blips 2 to 7 are quite similar with slightly higher current on the top. By adjusting the bias potential and the RF power differently between the top and bottom drivers the ITER requirement of beam uniformity $>90 \%$ is experimentally achievable. The divergence of this pulse is $\sim 2.7^{\circ}$ (measured by BES) with a ratio between acceleration voltage and extraction voltage of 4 . Beam divergences down to $1^{\circ}-1.5^{\circ}$ could be achieved at a ratio of 5-6. More details about beam uniformity

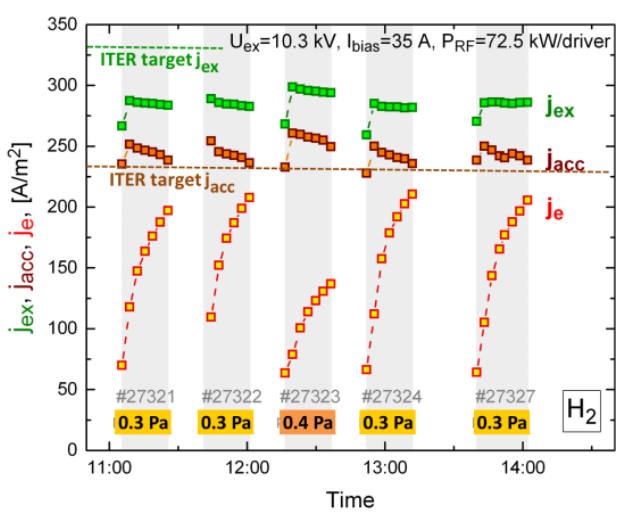

Fig. 4 [12]: Series of six 1000 s pulses with high performance. The $2^{\text {nd }}$ pulse has a pre-plasma phase of $200 \mathrm{~s}$, the third pulse is done at 0.4 Pa filling pressure.

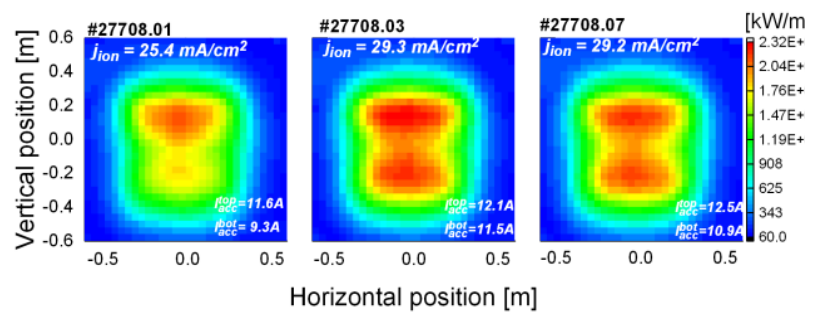

Fig. 5: Power density distribution of the beam on the diagnostic calorimeter for the best pulse (Fig. 3), left for the $1^{\text {st }}$ beam blip, middle for the $3^{\text {rd }}$, right for the $7^{\text {th }}$ beam blip.

measurements can be found in [14].

\section{Magnetic filter field variations}

The magnetic filter field of ELISE is generated by an electric current flowing vertically through the plasma grid (PG current) and back through three return conductors beside the driver exit. This solenoid-type coil forms a transversal magnetic field in the expansion chamber while the plasma generation in the drivers is in a lowfield zone and the beam region (downstream of the PG) is almost field free. The source performance could be improved at ELISE by adding permanent magnets (cross section $9 \times 39 \mathrm{~mm}^{2}$ ) along the side walls of the source, particularly for low pressure operation in D [9]. A typical magnetic field plot for hydrogen operation is shown in Fig. 6a using 1.5 kA PG current plus external magnets. The magnetic field strength scales with $\sim 1 \mathrm{mT} / \mathrm{kA} \mathrm{PG}$ current $+1 \mathrm{mT}$ external magnets in the center of the PG. In general higher magnetic fields of $\sim 4 \mathrm{mT}$ are required for deuterium operation.

The filter field for ITER and MITICA is designed without permanent magnets under the boundary condition to have $3 \mathrm{mT}$ in front of the $\mathrm{PG}$ and a long range field of $0.5 \mathrm{mT}$ at the exit of the accelerator, which is required to deflect secondary and stripped electrons out of the beam. This field topology is achieved by adding "side conductors" beside the source in the PG plane thus expanding the PG field sideward; their current is flowing back through the three return conductors beside the drivers (ELISE-like). The PG current itself is returned through two additional "remote return conductors" which are positioned far behind the source. The current ratio between side conductors and PG is fixed to $17 \%$ of the total current, namely $0.7 \mathrm{kA}$ out of $4.2 \mathrm{kA}$. The magnetic field design is described in detail in [15]. As the MITICA field has a different topology than the ELISE filter field (e.g. higher field in the drivers), simulating experiments have been performed on ELISE with side conductors and remote return conductors in the same position wrt the source as on MITICA (shown in Fig. 6b for reduced $\mathrm{I}_{\mathrm{PG}}$ ). Separate power supplies allowed for variation of the current ratio between them. Additionally external magnets could be installed or removed (Fig. 6c).

The experiments were performed in hydrogen with a well-conditioned source. Applying identical source parameters (pulse length $300 \mathrm{~s}$ with 3 beam blips, $\mathrm{P}_{\mathrm{RF}}=$ $67.5 \mathrm{~kW} /$ driver, $\mathrm{U}_{\mathrm{ex}}=10 \mathrm{kV}, \mathrm{U}_{\mathrm{acc}}=40 \mathrm{kV}, \mathrm{p}_{\text {fill }}=0.3 \mathrm{~Pa}$ ) the current through the PG $\left(\mathrm{I}_{\mathrm{PG}}\right)$ was adjusted in a way that similar ion current densities were achieved with the different FF. This was done with a fixed current fraction 

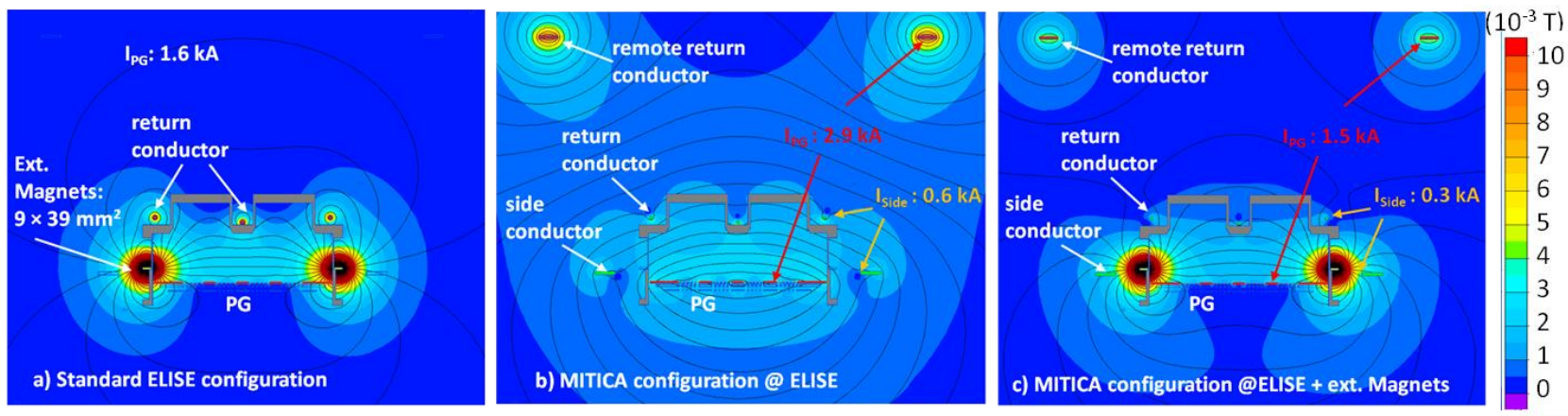

Fig. 6: 2D-magnetic field topology of ELISE (horizontal cross section), a) standard ELISE configuration with PG current \& external permanent magnets, b) MITICA configuration only PG current, c) MITICA configuration \& permanent magnets.

through the side conductors of $17 \%$, with and without external magnets in order to identify changes in the source performance, mainly the behavior of co-extracted electrons. Fig. 7 shows the influence on the current density of the co-extracted electrons and on their symmetry. For case $b$ (MITICA FF) the total PG current has to be increased by more than a factor of two to extract similar ion currents as case a, however, improving the situation significantly: the electron currents are reduced by $50 \%$ and almost symmetric, which is an important prerequisite for long pulses. For case c (MITICA FF + ext. magnets) the electron current is comparable to a but the symmetry is improved. It was further detected that increasing the MITICA FF to the ITER value of $4.2 \mathrm{kA}$ while keeping the current fraction through the side conductors constant at $17 \%$ reduces the amount of coextracted electrons further (by slightly decreasing their symmetry) but reduces also the extracted ion current by $\sim 20 \%$. Further optimization is possible by a flexible current distribution and adjusting the PG current for high negative ion yield while increasing the side conductor current to reduce the electron current and improve their symmetry. Although the overall outcome is very promising, it should be considered that due to time constraints these experiments have only identified trends in the source performance. Since any FF modification causes a different Cs redistribution, reconditioning over several

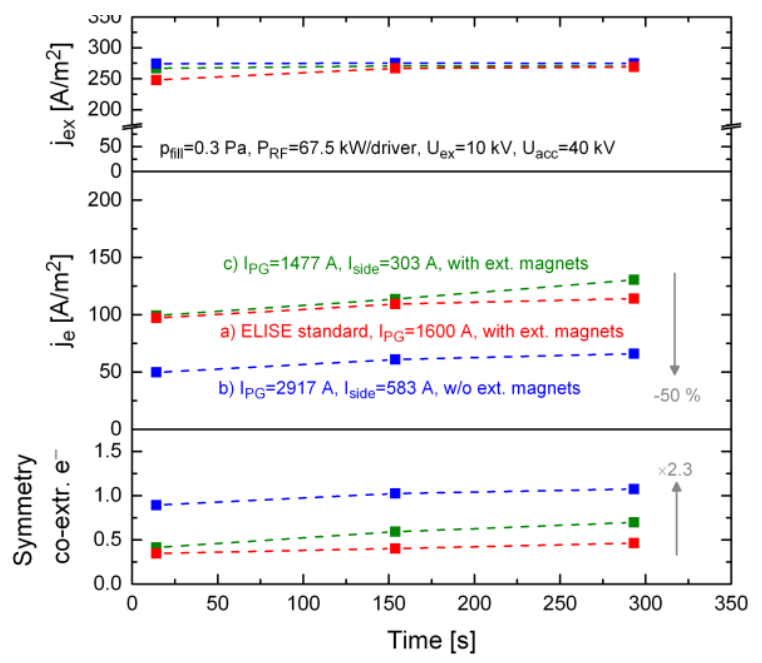

Fig. 7: Extracted ion current density (top), electron current density (middle) and electron current symmetry (bottom) for different magnetic filter fields: a) standard ELISE FF $\left(I_{P G}=1600\right.$ A \& external magnets), MITICA $F F$ w $/ o(b) \& w$ external magnets (c). $I_{P G}$ is adjusted for similar ion currents . days would be needed to assess long-term effects.

Experiments in deuterium could only be done very briefly showing the preliminary result that a PG current of $4.2 \mathrm{kA}$ is far too low to stabilize the electron current in long pulses. Permanent magnets seem to be mandatory.

\section{Summary}

In view of ITER's requirements for the RF source the test facility ELISE has achieved remarkable progress. Several technical source improvements and the use of vertical potential rods and external magnets enabled accelerated current density in hydrogen of $237 \mathrm{~A} / \mathrm{m}^{2}$ for $1000 \mathrm{~s}$. Also several long pulses could be performed in series during one day. For deuterium operation the applicable RF power and therefore the extracted negative ion current is still limited by the amount and temporal instability of the co-extracted electrons, which can also be very different on the top and bottom grid segments. Variations of magnetic filter field and electric potentials are promising tools for further improvement. A beam uniformity $>90 \%$ can be achieved by adjusting the RF power ratio between the drivers.

Tests at ELISE with the MITICA/ITER filter field indicate that this field configuration could lower the coextracted electron current and improve its top/bottom symmetry in hydrogen. For deuterium this might not be the case and further experiments are required.

\section{References}

[1] Hemsworth, R. S. et al. New J. Phys., 19, 025005, 2017.

[2] Kraus, W. et al. Rev. Sci. Inst., p. 02B104, 2012.

[3] Speth, E. et al. Nuclear Fusion, 46, p. S220, 2006.

[4] Toigo, V. et al. New J. Phys., v. 19, p. 085004, 2017.

[5] Nocentini, R. et al. Fus. Eng. Des., 86(6-8), 916-919, 2011.

[6] Heinemann, B. et al. New J. Phys., v. 19, p. 015001, 2017.

[7] Wünderlich, D. et al. Nuclear Fusion, 56, 106004, 2016.

[8] Heinemann, B. et al. Fus. Eng. Des., in press, https://doi.org/10.1016/j.fusengdes.2018.03.025, 2018.

[9] Wünderlich, D. et al. Plasma Phys. Control. Fusion, 58, 125005, 2016.

[10]Kraus, W. et al. Rev. Sci. Inst., 89, 052101; doi: 10.1063/1.5012591, 2018.

[11] Fantz, U. et al. AIP Conf.Proc. 1869, 030004, 2017.

[12]Wünderlich, D. et al. Long Pulse Operation at ELISE: Approaching the ITER Parameters, submitted to AIP Conf. Proc., 2018.

[13] Nocentini, R. et al. Fus. Eng. Des., 88, 913-917, 2013.

[14] Bonomo, F. et al. Uniformity of the Large Beam of ELISE during Cs Conditioning, subm. to AIP Conf. Proc., 2018.

[15] Chitarin, G. et al. AIP Conf. Proc., 1655, 040008, 2015. 